\title{
The ReflecTable: Bridging the Gap between Theory and Practice in Design Education
}

\author{
Jonathan Hook ${ }^{1}$, Thomas Hjermitslev ${ }^{2}$, Ole Sejer Iversen ${ }^{2}$, and Patrick Olivier ${ }^{1}$ \\ ${ }^{1}$ Culture Lab, Newcastle University, Newcastle upon Tyne, United Kingdom \\ ${ }^{2}$ Centre for Advanced Visualisation and Interaction, Aarhus, Denmark \\ \{jonathan.hook, patrick.olivier\}@ncl.ac.uk \\ \{thomashj, oiversen\} @ cs.au.dk
}

\begin{abstract}
The ReflecTable is a digital learning environment that explores how design games and video-led reflection might be combined to bridge the gap between the theoretical and practical components of design education. The concept seeks to leverage the qualities of exploratory design games and video to inspire design students to critically reflect upon the relationship between their evolving design practices and the theories and techniques they are taught in lectures, by allowing them to capture, review and reflect upon short videos of a design game. In this paper, we present the ReflecTable design and nine studies conducted during the course of its development. The studies suggest that the ReflecTable has the potential to support design students in understanding how theoretical concepts and methods relate to different design situations and their own evolving design practices.
\end{abstract}

Keywords: Design, design games, video-led reflection, education, reflection, reflective practicum, off-loop reflection, learning-by-doing.

\section{Introduction}

Educating students in the skilled and creative practice of design is a challenging task. Typically, the situations and challenges designers face are multifaceted, ill defined and, therefore, not easily addressed by the straightforward application of theories or guidelines [27, 29]. Consequently, it is widely accepted that approaches to educating designers that focus upon the teaching of theoretical concepts alone are unlikely to equip students with the skills required to respond appropriately to the challenges faced by design practitioners. Instead, design educators characteristically seek to train their students to dynamically adapt and employ a repertoire of theories, techniques and skills in response to their reflective appreciation of an individual design situation; a competency that Donald Schön refers to as a designer's "artistry" [31, p. 13].

The artistry of design practice is not something that can be easily taught, as it hinges on the student developing an understanding of how the particular theories and techniques that they have been shown in lectures and tutorials relate to their own personal experiences of designing in response to a range of different situations and challenges. Purely theoretical education has been found to be ineffectual at facilitating 
this kind of learning, as novice designers do not have the necessary experience of design practice in order to develop these contextual and holistic understandings of what they are taught [31, p. 82]. However, the alternative, a focus on practical education through design projects, has also been found to fall short, in our own and others' experiences. While learning-by-doing allows students to develop personal experience of design practice, students are often given little opportunity or motive to reflect upon the relationship between the theories and techniques they are taught and the situations they encounter during their design projects [37].

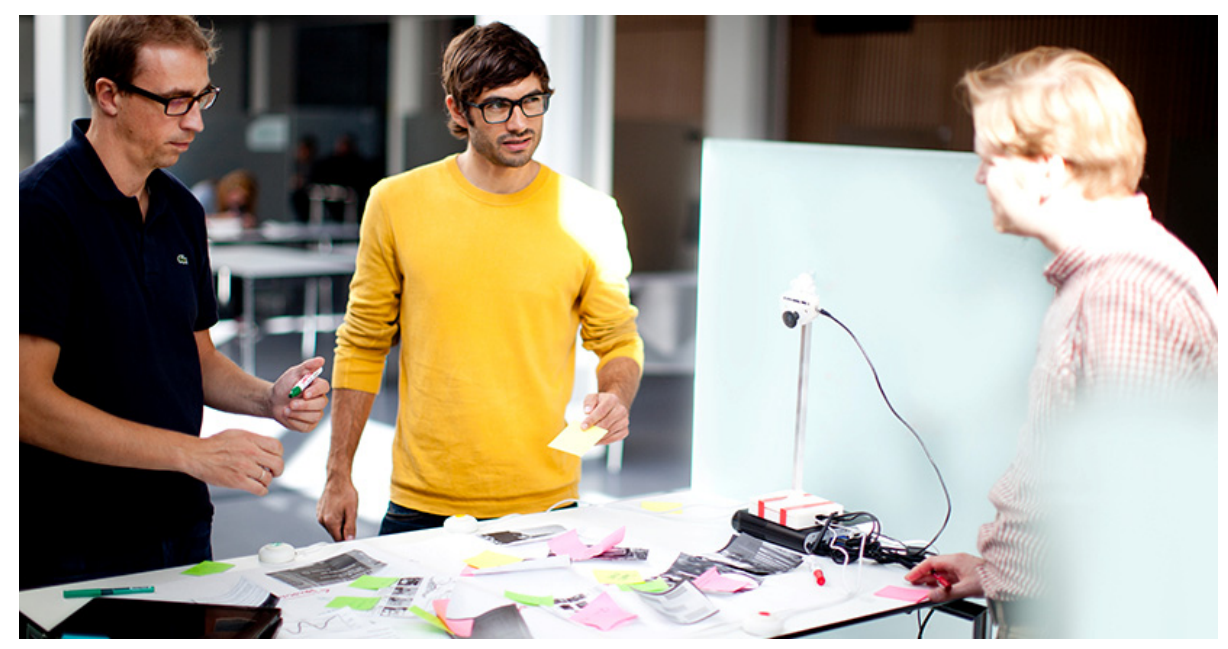

Fig. 1. The ReflecTable

Our response to this challenge, the ReflecTable (Figure 1), is a digital learning environment that seeks to support students in reflecting upon a condensed design process, which is completed while playing a group design game around a tabletop. Students playing the ReflecTable game are given a number of small buttons that can be pressed to capture short video clips of noteworthy moments in their design process. Following the completion of this game, the students are guided through a session of structured reflection on their design process, which involves reviewing and annotating the video clips captured using a bespoke tablet PC application. By leveraging qualities of both design games and video-led reflection, the ReflecTable seeks to provide design students with an environment that combines learning by doing with critical reflection about the relationship between design practice and theory.

In the remainder of this paper, we review previous work relating to the use of digital technology to support design practice and education, describe the design of the ReflecTable in detail and, finally, reflect upon our initial experiences of using the ReflecTable as part of the education of undergraduate design students. These experiences illustrate the design's potential as a means to support students in reflecting upon and, therefore, developing further understandings of the relationship between their evolving design practices and the design vocabulary and procedures (i.e. theoretical components) that they are taught as part of their education. 


\section{Related Work}

\subsection{Interactive Systems for Design Practice and Education}

The use of digital technology to support design practice and education can be seen to be an important concern of the HCI research community. A range of previous research has led to the development of a number of novel interactive systems that support both the practices of designers and the education of design students.

Several interfaces have been developed by HCI researchers that seek to enhance the existing practices of designers through the application of digital technology. A number of hybrid interactive surfaces [20] have been proposed that aim to seamlessly integrate the workflows and physical artifacts of the traditional design studio with advantages of digital technology. These interfaces have used approaches such as computer vision $[17,21]$, tangibles and digital pen and paper technology $[14,15]$ to digitally augment the physical artifacts used during traditional design meetings (e.g. post-it notes) with functionality such as saving and versioning and support for the transition to later, digital, stages of the design process. Additionally, a number of designs have been proposed to afford lightweight integration of digital technology and digital media into design meetings [33,34] and the configurations of artifacts and space witnessed in the designer's traditional studio [1].

HCI researchers have also explored the development of interfaces that enhance collaboration and awareness amongst design teams. The Cooperative Artifact Memory system was developed to allow designers to share digital information about artifacts from a design process with their collaborators such as annotations, messages and web-links using a mobile phone application [38]. Blevis et al. developed an interface that aimed to scaffold collaborative decision making in respect to both physical and digital design materials, which were associated together using barcodes [2]. Additionally, work by Everitt et al. explored how the use of hybrid interactive surfaces (such as those mentioned previously) might support collaboration amongst designers in different geographic locations [10].

Of most relevance to the ReflecTable, however, are previous research projects that have explored how digital technologies might support designers, and design students, in documenting and reflecting on their design processes and practices. Nakakoji et al. proposed a tool that indexes videos of design meetings based upon sketches drawn on a whiteboard. Their system aimed to allow designers joining existing projects and teams to more easily use videos of design meetings to develop understandings of, and reflect upon, design decisions made previously by the existing design team members [25]. Geyer and Reiterer explored the use of the commercial note taking software Evernote to support design students documenting longitudinal design projects [13]. Swan, Tanase and Taylor explored the Digital Scrapbook system, which automatically collects digital materials from, e.g., design students' blogs and Flickr pages [35]. In both of these studies, providing students with a mechanism to collate, document and review design project materials was found to inspire and support reflection on their design practices. In the latter case, the students' reflection on their practices using the Digital Scrapbook system was found to make students aware of the 
"Processional" nature of the design processes they conducted. Additionally, in the context of design research [39], Dalsgaard and Halskov developed the Project Reflection Tool, which allows design researchers to document their practices as a series of events and notes, with the aim of supporting reflection on both design practice and broader research questions [8].

A number of research projects have looked beyond the possibility of simply supporting the documentation of design processes, to explore instead how the designer's active manipulation of digital design materials might inspire reflection on design practice. The Amplifying Representational Talkback system aimed to inspire reflection during the design process by allowing designers to experiment with different two-dimensional arrangements of their digital design materials [26]. Additionally, the Freed system enabled design students to create multiple spatial representations of the collections of digital materials that are amassed during longitudinal design projects. This process was found to inspire design students to reflect on their design practices throughout the course of a design project, rather than simply upon its completion [24].

\subsection{Using Design Games and Video to Inspire Reflection}

In the following sections, we review a range of additional work on design games and the use of video as a means to inspire reflection, which influenced the ReflecTable design. The idea of playing games to study design practice derives from research in architecture and planning from the late eighties. At MIT, Harbraken and Gross [16] developed Concept Design Games to study how design decisions and choices are made in a process of cooperation and negotiation among multiple stakeholders in interdisciplinary design projects. By identifying similarities between design collaboration and simple board games, Harbraken and Gross envisioned their own board games that isolated and focused on certain aspects of the design process. Games as a method to understand design was further developed in Participatory Design literature as a way to understand user collaboration in design [9], to support design practitioners in their collaborative design processes [18] and to develop a repertoire of gaming possibilities for designers to be aware of when creating their own exploratory design games $[3,4]$.

To some extent, games have also been used in design education. Buccerelli [6] identified gaming as a way to teach engineering design students about different roles in the design process. Iversen et al. discussed the educational benefits of design games [18]. They envisioned design games as a way to train design collaboration in a "safe" environment. With respect to this safe environment for design team training, design games have similarities with the notion of "Serious Play" developed by LEGO, where LEGO bricks are used to explore real life challenges in business development [28].

The value of design games as a means to support design education has, however, been questioned in recent Participatory Design literature. Törpel critically argued that what students learn from a design game might be limited by the different scales of students' design projects and real life design practice: "What in relatively small education projects can be experienced on a small-scale level could become substantial 
factors of success or drawback if practiced on a larger scale in professional design projects" [36]. This challenge has been addressed by combining design games with video material from real life design situations. Buur and Søndergaard [7] developed an augmented board game in which video from real life design work was made accessible to the participants. In this augmented space, qualities from the design game were combined with real life design situations captured on video and brought into the design game using augmented paper sticks. Iversen \& Buur [19] developed a Video Design Case in which design practitioners could reflect on their own design skills by collaboratively working with video clips from an industrial design case. The Video Design Case made design practitioners reflect on their own practice by mirroring their own design with the design activities displayed in the case material. Iversen \& Buur found that video is a valuable approach for fostering reflection on design practice; however, the extent to which designers can familiarize themselves with videos of other designers' practices and cases may limit the reflection that can be spurred by the approach.

The ReflecTable extends this existing body of research by exploring how a novel system for video-led reflection on design practice might be combined with a gaming approach to reflection on design to address the particular challenge of bridging the gap between the theoretical and practical components of design education.

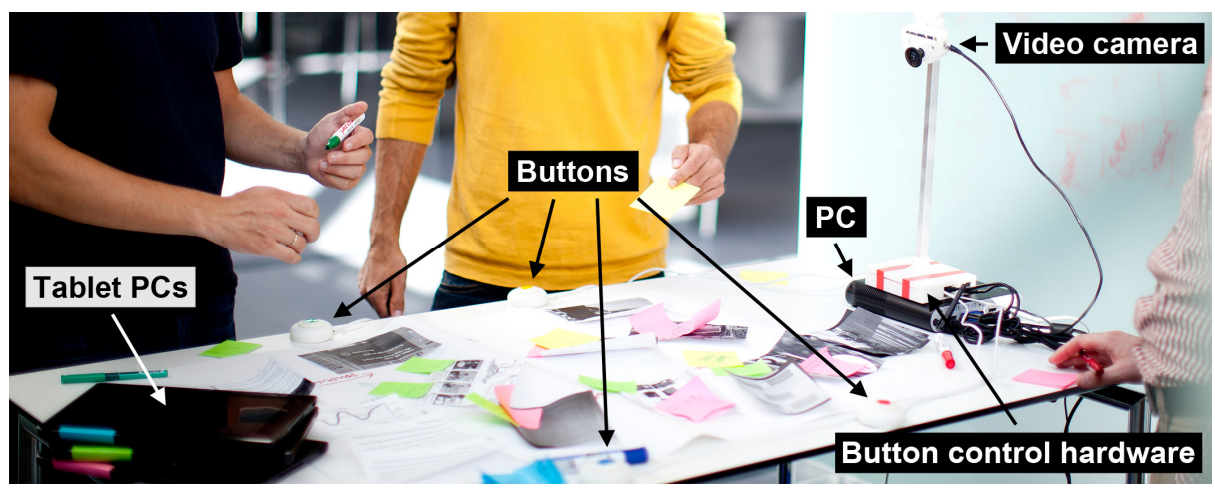

Fig. 2. The key components of the ReflecTable system

\section{The ReflecTable}

The ReflecTable is a digital learning environment that seeks to leverage qualities of both design games and video as means to inspire designs students to critically reflect upon the relationship between their evolving design practices and the theories and techniques they are taught in lectures. This is done by allowing them to capture, review and reflect upon short videos of their own design process in a design game.

The ReflecTable (Figure 2) comprises three key elements: a paper-based design game that is played around a tabletop between groups of three to four design students, a video capture system that allows students to capture short video clips of noteworthy moments during their game by pressing a number of buttons and a tablet-based 
interface that allows students to review and reflect upon these videos upon the conclusion of the game.

The design game is comprised of two stages and six rounds (see Table 1 for an overview) and is played around a tabletop between groups of three to four design students. This game moves between divergence, utilizing turn taking inspired by Harbraken and Gross' silent game [17], together with convergence to emulate the phases of a real life design project [22]. In the following sections we describe the course of a typical ReflecTable session.

Table 1. An overview of the rounds of the design game

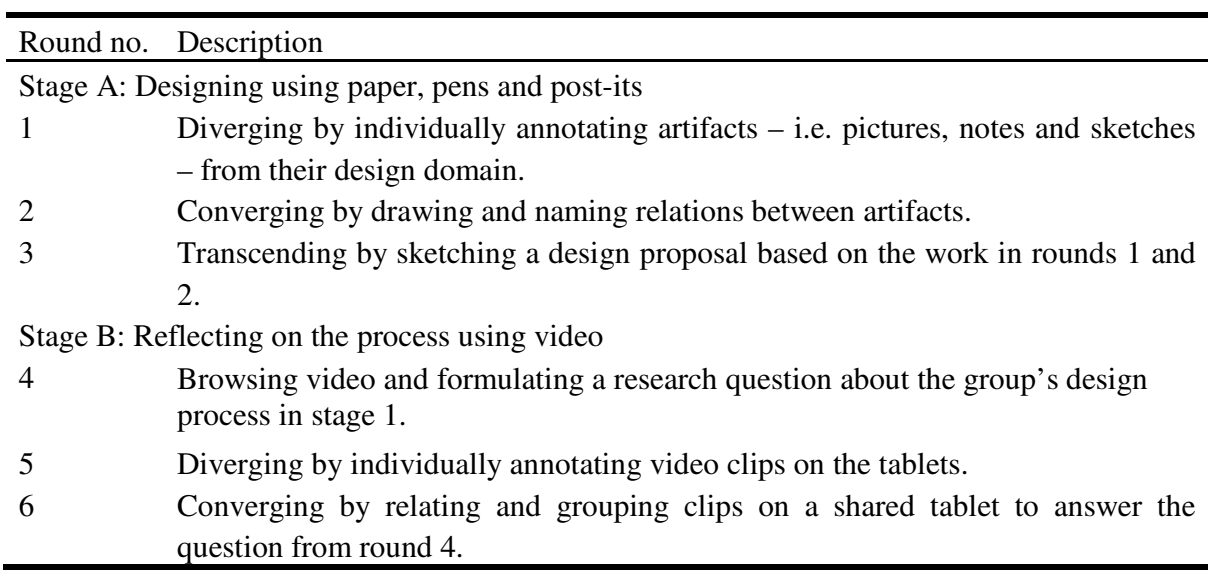

\subsection{Stage A: Designing on Paper}

The aim of the first half of the design game is to co-develop a design in response to a design situation shared by the players (e.g. a brief set during a group project). The stage comprises three rounds, which each mimic a phase in a condensed design process. A game facilitator guides the group throughout the game, offering coaching to the players if, for instance, they become stuck, or need further advice.

During this first half, a video camera is used to record an image of the table surface and a microphone records the discussion between players and the facilitator. Each player is given a button, which can be pressed at any time during the game, to record the previous 15 seconds of video. The players are instructed to press this button when something noteworthy has happened. For instance, a player might press their button when a particularly interesting idea arises or when s/he feels that something is worth remembering.

The group is asked to bring five artifacts (per player) to the game, which must relate to the design situation and be small enough to be placed and manipulated on the tabletop. In our experiments so far, people have brought a wide range of different artifacts including pictures from field studies, vision statements and scenario descriptions. 
In the first round, the group is instructed to place their artifacts on the table for individual exploration by each of the players. The players are given post-it notes (a different color for each player) and are asked to write comments about any of the artifacts that they find to be in any way remarkable. At the end of the round, the players present these annotations to each other. The purpose of this round is to allow players to develop divergent interpretations of the artifacts, which will be used as the basis of a shared understanding of the design situation developed in later rounds. Consequently, the players are asked to complete this task without communicating with the other players. The players are given about 10 minutes to complete this round.

In the second round, the players are asked to collaboratively name and draw relationships between the artifacts, which have now been augmented with post-its. The players are instructed to arrange the artifacts and their comments on the table surface, so that they illustrate relationships and shared understandings of the materials and design situation developed by the group. The purpose of this round is to converge the players' individual interpretations of the design situation into a shared understanding, upon which they might base a design. The players are given about 20 minutes to complete this round.

The third round of the game involves transcending the ideas and relations discussed in the previous round, in order to propose a concrete design response. Firstly, the players are asked to collaborate in identifying three potential design openings, i.e., places in the network of relations between artifacts and notes that they feel have the potential to be the starting point for a promising design. Then, the players are given exactly 15 minutes to sketch and write-up (informally) a proposal for a concrete design response to the situation, based upon the ideas developed during the previous rounds.

\subsection{Stage B: Structured Reflection on the Design Process}

In the second half of the game, a tablet-based interface is used to scaffold reflection amongst the players about the design process conducted during the first stage. Each of the players is given a tablet PC (Acer Iconia Tab W500) with a bespoke interface that allows them to review, annotate and, finally, collate and compare the videos captured by pressing the buttons during the design game. Like the first stage, the second stage is structured around three rounds. The facilitator offers coaching and guidance throughout the three rounds.

In the fourth round, the first round of stage B, the interface displays a Panopticon video rendering, which allows the players to quickly view footage of the entire design game at a glance (Figure 3). This Panopticon rendering works by dividing a video into a number of clips of equal length, which are then arranged in a grid sequentially from left to right, (akin to a cartoon strip). All of these clips are played concurrently in a continuous loop. As they play, the videos slowly cycle towards the right, such that when a video has reached the end of its loop it is in the starting position of the video to its right. This movement of video clips allows the viewer to watch the video continuously, from a selected point, by simply following the passage of one square in the grid of videos. 
This Panopticon video is overlaid with a number of color-coded markers, which each highlight a point in the video where a particular player pressed their button in order to indicate a noteworthy event. The players are able to touch these markers to view a larger version of the 15 seconds of video recorded before that particular button press, which is presented, with sound, in a pop-up window. Using this interface, the players are asked to collaboratively review the video clips created during the first stage. While reviewing these video clips, the players are asked to formulate a research question about their design process that they would like to explore during the remainder of the ReflecTable game. In the studies we have conducted so far, players have sought to answer questions such as "what in the process made us change direction?" and "can you see traces of our current design in this workshop?"

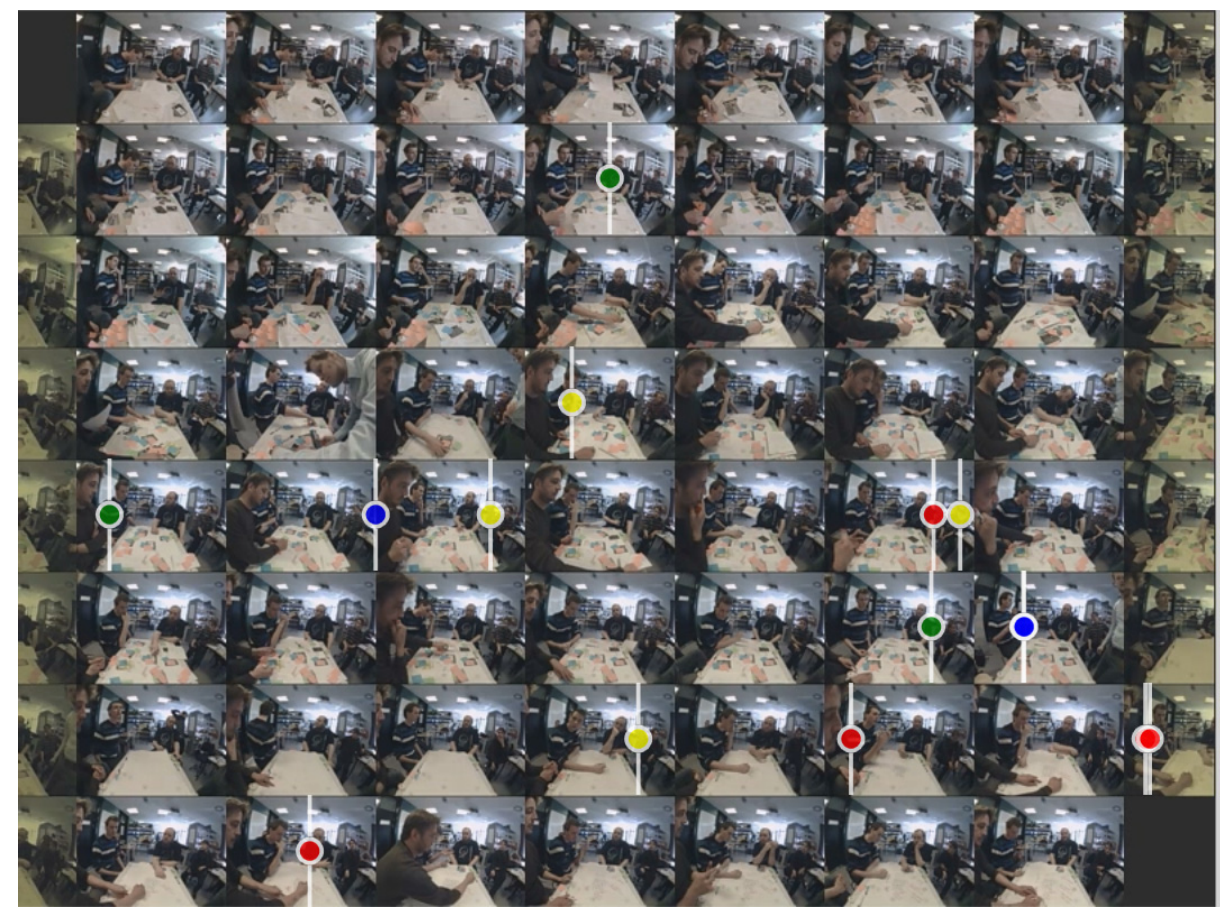

Fig. 3. A Panopticon video of a design game

In the fifth round, the participants begin to explore the video clips from the design game, with the aim of answering their research question. During this round, the tablet interface is augmented with an additional text box and virtual keyboard, which enables the players to annotate selected video clips. Each player is asked to choose three video clips that offer insight into their research question, using the annotation functionality to note the rationale for each selection. As the purpose of this round is to allow the players to propose potentially divergent interpretations of each of the video clips, this annotation process is completed individually, with players coming together at the end of the round to present their selections to the other group members. 
During the final round, the players collaborate around one of the tablets, to develop an answer to their research question by drawing and naming relations between the clips annotated in the previous round. The single tablet displays an interface (Figure 4) that shows each of the clips, surrounded by any annotations made by the players. Annotations are represented by virtual post-it notes, which are colored in order to identify the player who wrote them. These annotations, and the videos to which they relate, can be moved, rotated and resized on the interface using simple multi-touch gestures. Furthermore, a mode can be selected whereby the players are able to write and draw on the background of the screen, to illustrate connections between particular clips and annotations. This final activity seeks to scaffold the players in converging their individual understandings of the clips into an answer to their research question, which in turn represents a shared understanding of the design process conducted by the group.

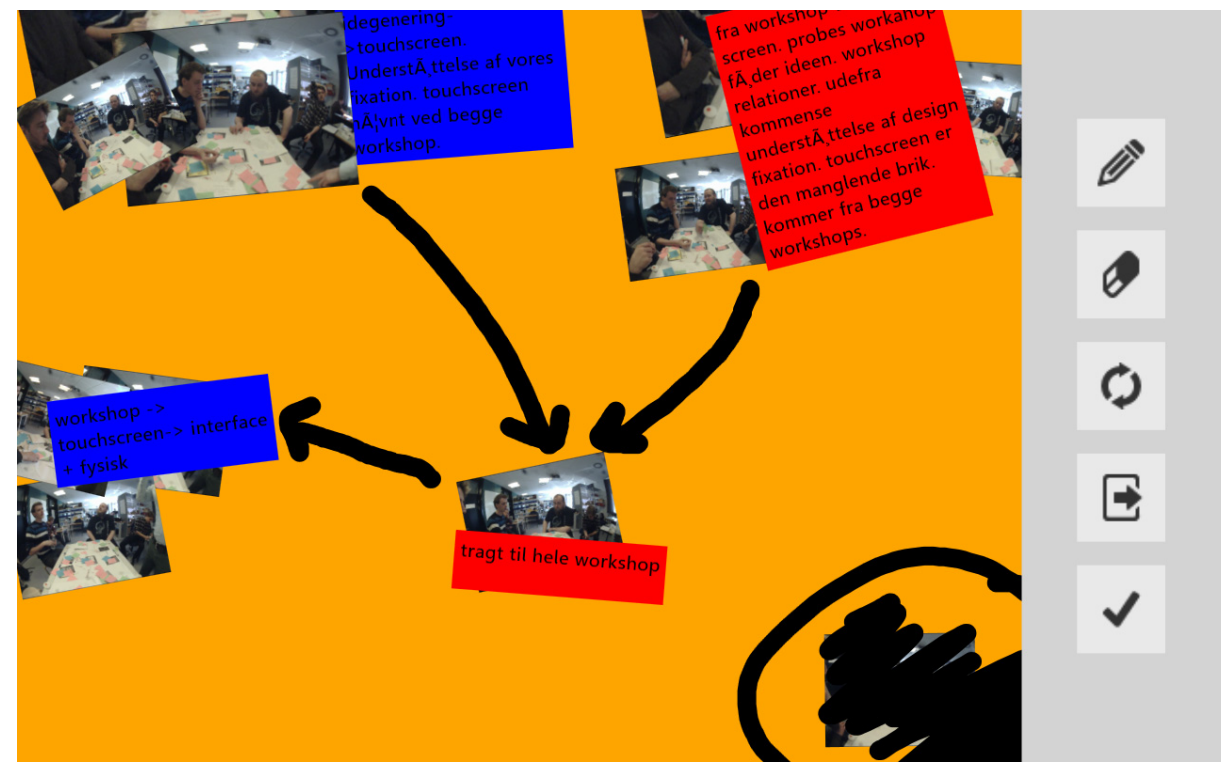

Fig. 4. The interface used in the last round of the game

\section{Development and Exploratory Study}

The ReflecTable was designed to explore how design games and video-led reflection might be combined to bridge the gap between theory and practice in design education. We used a research-through-design approach [39], whereby the iterative design and development of the ReflecTable game and hardware platform was used to scaffold our investigation of this research question. This design and development process occurred over the course of nine ReflecTable games, which were each played with three to four players. In the first six of these games, we focused on developing the game rules using a mock-up of the final setup. In the final three games, we tested the 
finished prototype of the ReflecTable setup, as described in the previous section. We documented each of these games using video, pictures and observational notes.

In order to explore if the evolving design game was supportive of students' reflection upon the relationships between design theory and practice, before commencing development of the final prototype, we studied two of the initial six games in further detail (games 2 and 3 in the series). As these games were played prior to the development of the final prototype, we used a mock-up system that allowed students to press buttons to record video clips. However, manual video editing was required to produce the video media for the second stage of the game. This meant that two time slots had to be scheduled and, as such, the two stages of the game were played two to three weeks apart. In the following sections, we describe the findings of this exploratory study. We present these initial exploratory findings as they provide valuable insight into the kinds of learning supported by the ReflecTable game, which was not changed substantially from the version used in this study to the final design.

The study was completed with two groups of undergraduate interaction design students, who were in their first semester. The design education of these students had been based primarily upon the work of Schön on reflective design practice $[30,32]$ as well as the theories of Löwgreen and Stolterman [22], and Nelson and Stolterman [27]. To explore the learning outcomes from these two games, we gathered two kinds of qualitative data from the participants. On completion of stage B, we asked the participants to write three post-its about the group's outcome and present these to the other players. We then conducted short semi-structured interviews [12] (six minutes on average) with each participant about their experiences of playing the game and what they thought they had learned. These interviews included a targeted question that sought to explore whether the students could relate their experiences of playing the ReflecTable game with concepts from Schön's theories of design. By asking the students to comment upon the relationship between their design process and one of the theories they had been taught, our intention was to gain insight into whether the game had supported them in learning to relate their experiences of practice to design theory. The questions asked were as follows:

1. What did you personally gain from playing the game?

2. What did you learn about designing by playing the game?

3. Can you recognize any of Schön's concepts in the game?

4. What do you think our intention is with this game?

We recorded and transcribed these interviews and, subsequently, analyzed them using two different coding procedures ${ }^{1}$. Firstly, an inductive approach was used, where we coded and grouped students' comments into themes and, secondly, a theoretical approach was used where we identified terms from design theory primarily Schön's theory - in the students' responses [5]. Table 2 shows an overview of the design concepts that we identified in the interviews as either an explicit

\footnotetext{
${ }^{1}$ The excerpts presented from these transcripts have been translated from Danish to English.
} 
Table 2. Design theory concepts identified in the interviews

\begin{tabular}{|c|c|c|c|c|c|c|c|c|}
\hline \multirow[t]{2}{*}{ Design terms } & \multicolumn{3}{|c|}{ Players } & \multirow[b]{2}{*}{$2-4$} & \multirow[b]{2}{*}{$3-1$} & \multirow{3}{*}{$3-2$} & \multirow[b]{2}{*}{$3-3$} & \multirow[b]{2}{*}{$3-4$} \\
\hline & $2-1$ & $2-2$ & $2-3$ & & & & & \\
\hline Conversation with the materials & & A & & $\mathrm{A}(\mathrm{x})$ & B & & & A \\
\hline Design judgment & & A & & B & & B & B & \\
\hline Learning-by-doing & A & & A & A & A & B & & A \\
\hline Professional artistry & A & & & & $\mathrm{A}(\mathrm{x})$ & & & \\
\hline Reflection-in/on-action & & & $\mathrm{A}(\mathrm{x})$ & $\mathrm{A}(\mathrm{x})$ & $\mathrm{A}(\mathrm{x})$ & & $\mathrm{A}(\mathrm{x})$ & $\mathrm{A}(\mathrm{x})$ \\
\hline Reflective practice & & & & & A & $\mathrm{B}(\mathrm{x})$ & & A \\
\hline Repertoire & & & B & B & A & & B & A \\
\hline World-making & & & & & & & $\mathrm{A}(\mathrm{x})$ & $\mathrm{A}(\mathrm{x})$ \\
\hline
\end{tabular}

statement (marked with ' $(\mathrm{x})$ ') or an implicit description. An 'A' indicates that a concept was mentioned after the topic of Schön's theory had been broached in discussion (e.g. question three had been asked), while a ' $\mathrm{B}$ ' is used where the players mentioned concepts beforehand.

\subsection{Learning Outcomes}

Schön notes that it is hard to know exactly whether a student has been taught the "artistry" of design (i.e. the skill of being able to understand and apply a repertoire of theories and approaches sensitively and appropriately in practice) that the ReflecTable seeks to teach. However, he describes four dimensions of learning [31, pp. 168-170] that represent competencies that a student might possess in varying degrees. In the following sections, we discuss the students' responses to the semi-structured interviews in terms of these dimensions. We refer to the students using a system where the first number denotes the game/group and the second the player (e.g. "player 2-4" or "player 3-2").

Understanding Vocabulary. The first dimension that Schön described concerns the student's ability to know and use the vocabulary of their field. The student should not only be able to name the concepts that they have been taught, but demonstrate an understanding of the processes to which they refer and their relationship with design practice. The results of the study suggest that the ReflecTable can help students to gain a better understanding of the relation between the design vocabulary that they knew from lectures and their own design practices. In the interviews, all of the participants described how they had encountered one or more design concepts during the game. Table 2 illustrates how some students, such as player 3-2, were very good at using the vocabulary offhand, while others, such as player 3-4, were very good at identifying the concepts when prompted to do so.

The most prominent design concepts that students demonstrated an understanding of when discussing the ReflecTable game were those of reflective conversation with materials and learning-by-doing. For example, player 3-1 discovered that designing is easier when you work actively with the artifacts or, in Schön's words, have a "reflective conversation with the materials of the design situation". 
Researcher: What did you personally gain from participating in this design game? Player 3-1: Well, I probably realized, that I'm better at thinking creatively when I'm doing something [...] Because I've sometimes wondered 'Am I even thinking creatively enough' and stuff like that, when you're sitting by your computer and 'now, you should write a design proposal'. And then you try something like this, and you feel that, 'yes, you can do it'.

These are important insights because the metaphor of conversation embodies some of the most central pragmatic points in both Schön's theory of design and many of the other theories that the students are taught as part of their course. We believe that the students' ability to externalize connections between their practice and design theory in this way suggests a stronger understanding of the design vocabulary and its connection to design practice, as a result of playing the ReflecTable game.

Appropriating Procedures. The second dimension concerns a student's ability to apply design procedures sensitively and appropriately. Schön states that a competent student should not only know how to apply discrete techniques, but also to combine them and tailor them to the specific demands of a design situation. Every designer has what Schön terms a "repertoire" of design techniques, which they can apply appropriately and fluidly in their practice. Interestingly, students were spurred to reflect upon the role that the ReflecTable game itself, as a design method or technique, might play in their wider and future design practices. Five of the students described how particular aspects of the design game (e.g. the convergence and divergence of ideas in the group) could be put in their toolbox for later use. For example, one student explained that he felt his group could appropriate aspects of the ReflecTable game for use in their future design practices.

Player 3-3: All this you have shown us here is naturally a part of your own project, but it is also something that we can take parts of and take them out of their context, break them down a bit and use them as a tool in themselves. For example, we take a lot of pictures and group them, kind of like a new twist on the inspiration card workshop (see [23]) that we already know.

Students were able to highlight a range of qualities of the ReflecTable design game that might be appropriated in their future practices. As for the ReflecTable itself, three of the four students in group 2 commented on the value of ReflecTable as a learningby-doing educational tool, while group 3 saw it as an idea generator because the process of playing and reflecting upon the game had helped them to come up with a new idea for their project.

Generalizing Cases and Critical Stance. The third dimension describes a student's ability to generalize their experiences of design practices, as insight that will inform how they address future design situations, while the fourth dimension concerns a student's ability to critically appraise the traditions of the field to which they are being inducted. The students did not clearly exhibit such competencies when playing 
the ReflecTable game during our studies. However, we hypothesize that this might be because the students had only just begun their education and, as such, had limited practical and theoretical knowledge upon which to base discussions, reflections and critical evaluations of theory. We expect that more experienced students would have been better equipped to tackle these higher order issues when playing the ReflecTable game.

\section{Discussion: Scaffolding Reflection on Design}

The ReflecTable was designed to bridge the gap between the theoretical and practical components of design education, by stimulating design students to reflect upon a condensed design process conducted during a design game. Our initial studies of the ReflecTable suggested that it supports design students in understanding how the theoretical concepts (vocabulary) and methods (procedures) they are taught in lectures relate to different design situations and their own evolving design practices. In this section, we reflect further upon our experiences of using the ReflecTable throughout the course of all of the nine design games (including three using the final prototype) to examine how the different components of the ReflecTable contributed to the students' reflection on design practice. Consequently, we uncover insights that will guide and inform those wishing to design future interfaces that support reflection in design education and, potentially, other related domains.

\subsection{Using 'Real Life' Design Artifacts and Situations}

The ReflecTable game used artifacts and materials from real design challenges and situations faced by the players. These artifacts were found to form the basis of much of the discussion amongst participants, by representing and highlighting different perspectives and experiences upon the shared design problem. We found that the incorporation of real life materials and situations into the learning environment heightened the players' engagement with the game because they had a real stake in the project they were working with. Conversely, in situations where participants had already completed the idea generation phase of their project, we found that they tended to be less engaged with the game as its outcome would have a lesser affect on their work.

Most crucially, the use of artifacts drawn from real design projects, allowed students to reflect upon aspects of their design processes, which might not have otherwise been broached in the limited setting of the design game. For instance, artifacts from field studies served to ground the game in an actual design setting, highlighting issues that might have been overlooked had the game been framed as a simple discussion of the problem at hand. Consequently, we argue that the use of real life artifacts and design situations proved to be an invaluable quality of the ReflecTable, as it situated the design game, and subsequent video-led reflection, in the context of the players ongoing, real life design practice. Törpel [36] has argued that the educational value of design games might be limited by the difference between 
experiences developed while playing design games and those required of real world design practice. We believe that our use of students' own real life materials and situations to foster reflection on wider design practice, rather than just the condensed design process completed during the session, stands out as a potential way to rectify this potential limitation of design games.

\subsection{The Buttons}

Players were given buttons that could be pressed during the ReflecTable game, in order to capture the previous 15 seconds of video, for review in the second stage. By providing this lightweight video-capture system, our intention was to build upon previous work that leverages design documentation as a spur for reflection on the design process, by allowing design students to capture video of actual momentary experiences of designing for later reflection, in addition to artifacts and notes. Furthermore, by providing a mechanism to capture noteworthy moments in the design game, we sought to frame stage A itself as a reflective process, rather than simply an activity to be reflected upon during stage B. That is to say, the simple action of pressing the buttons was designed to inspire the students to engage in a higher form of reflection about what was interesting and important about their design process, while it was still ongoing.

Throughout the studies, the students were found to use the buttons as we intended to mark moments that they wished to remember and discuss later in the session. Often, button presses would occur collaboratively, with a press proceeded by a discussion of the relative interest of an event. In some cases, the players' use of the buttons took on an unexpectedly performative quality, as the visible pressing of a button acted as an expression to the remainder of the group that an interesting or important event had occurred.

Schön describes "reflection-in-action" (i.e. reflection which occurs during the course of an activity such as designing) as often being spurred by an unexpected or surprising interruption of a normally automatic activity [32, p. 56]. We observed that the simple task of pressing a button when a noteworthy event occurred, and observing and discussing the button presses of others, interrupted the design process in such a way that scaffolded both individual and collective reflection-in-action. Therefore, we hypothesize that the buttons might have acted as more than simply a means to record video clips of noteworthy events from the design game, as the source material for later reflection, but rather, were an important stimulus for the students' reflection on the relationship between theoretical and practical aspects of design observed during the studies.

\subsection{Video-Led Reflection}

In the second stage of the ReflecTable game, the students were asked to formulate and explore a research question about their design practice, using the video clips captured of the condensed design process conducted during the previous stage. These clips were explored using a Panopticon video rendering, which allowed the players to see 
video footage of the moments surrounding each button press, the temporal relationships between button presses and, finally, a video overview of the whole design game, all at a glance on one interface. Consequently, we intended to provide the players with a powerful tool that allowed them to not only browse and view individual video clips, but also to evaluate, discuss and reflect upon them in the wider context of the whole design game.

Reviewing video clips in this way was found to offer participants a practical way to discuss each button press in the detailed context within which it occurred; therefore, fostering situated and holistic consideration of the key moments in the design process. By combining this post hoc reflection with the reflection-in-action fostered during the initial stage of the design game, the ReflecTable extends previous work that has sought to support designers in reflecting either in $[24,26]$ or on $[13,25,35]$ the design process, but not both. We found that this combination of reflection in and on action, as part of a single design game, led to a valuable two stage reflective process that allowed participants to revisit initial moments of reflection (spurred by both playing the design game and the pressing of buttons) for further detailed and prolonged examination. Furthermore, by allowing students to reflect upon videos of their own practices, the ReflecTable also responded to the concerns of Iversen and Burr [19] that designers might struggle to empathize with, and hence reflect upon, videos of other designers and design cases that they are not involved in.

The participants who tried the prototype setup were generally enthusiastic about the potential of the Panopticon video rendering. However, we found that the Panopticon experience was limited by the horizontal camera position, which prevented participants from using it to examine the materials on the table in detail (i.e. to read the contents of post-it notes). We believe that using a top down camera might have rectified this problem. Furthermore, the initial design of the interface only allowed players to view the video clips captured during the session in high resolution. Therefore, players could not explore the context of a button press in detail, beyond the initial 15 seconds of video captured. While this issue could be resolved easily by allowing students to use the Panopticon rendering to explore the entire video as well as button presses, it is anticipated that such changes to the design might dilute the meaning of a button press during the design game and, therefore, its ability to provoke reflection amongst players.

\subsection{The Frame and the Facilitator}

The facilitator played a central role in the ReflecTable, guiding the students through the game, explaining the rules, answering questions and encouraging the players when they became stuck. The students found that, in these ways, the facilitator played an important role in supporting their learning during the game. For example, player 2-4 described the value of the "gentle provocations" that the facilitator gave to the group during the session, which, when combined with the frame of the design game, guided the players to a successful outcome. 
Player 2-4: ... it is like 'now, you read some texts' and then we have some practice classes, where we have Stine [an older student] walking around and coaching a bit. But she only comes around to the group once and she is does not stand by us and provokes us gently and sets up some frames [for the group work].

The idea that the facilitator can observe the players designing, while playing the ReflecTable game, and interject to provide such gentle provocations (i.e. guidance and advice) highlights potential parallels between the facilitator's role during a ReflecTable session and Schön's notion of a coach. Schön described how the relationship between student and coach depends on an environment where the student "tries to do what she seeks to learn and thereby reveals what she understands or misunderstands", so that the coach might respond with appropriate guidance [31, p. 162]. By fostering reflective discussion amongst players about the design process conducted, the ReflecTable was found to push students to externalize their understandings of design practice. Consequently, the ReflecTable was found to act as a valuable mechanism to support the facilitator in appreciating both individual players' and whole groups' understandings of particular theoretical concepts; therefore, supporting his ability to provide informed and appropriate coaching to the students during the game.

However, we became concerned during the studies that the facilitator might have had too strong of an influence on the students' design practices. That is to say, we were concerned that rather than freely and intuitively applying their design practices during the game, students might have been preoccupied with living up to their perceived expectations of the facilitator (i.e. following their advice and the rules of the game unquestionably). For example, one player commented "Take a pen; I think he [the facilitator] would like you too". Therefore, we argue the presence of the facilitator, as coach, might in the case of some students actually prevent them from becoming immersed in the processes of reflection on design practice that the ReflecTable seeks to inspire.

\section{Conclusions}

In this paper, we have explored how the design of a digital learning environment might bridge the gap between the theoretical and practical components of design education. Our response to this challenge, the ReflecTable, builds upon previous work by combining video-led reflections and design work in a game set up, which enables students to comprehend both design theory and design practice in an interplay between understanding and doing design. The results of an initial exploratory study of the ReflecTable suggest that it has the potential to support design students in understanding how theoretical concepts and methods relate to different design situations and their own evolving design practices. Furthermore, our findings illustrate a range of valuable qualities of the ReflecTable design that might be drawn upon when developing future tools (e.g. the value of video as a means to combine both reflection-in and -on the design process).

In the future, we intend to extend this research by conducting a series of more extensive studies that compare the ReflecTable with other approaches to educating 
design students. We believe that such studies will help us better ascertain the effectiveness of both the ReflecTable and the combination of games and videoreflection as means to support design education.

\section{References}

1. Binder, T., De Michelis, G., Gervautz, M., Jacucci, G., Matkovic, K., Psik, T., Wagner, I.: Supporting Configurability in a Mixed-Media Environment for Design Students. Pers. Ubiquit. Comput. 8, 310-325 (2004)

2. Blevis, E., Lim, Y., Ozakca, M., Aneja, S.: Designing Interactivity for the Specific Context of Designerly Collaborations. In: Ext. Abs. of CHI 2005, pp. 1216-1219. ACM (2005)

3. Brandt, E., Messeter, J.: Facilitating collaboration through design games. In: Proc. of PDC 2004, pp. 121-131. ACM (2004)

4. Brandt, E.: Designing exploratory design games: a framework for participation in Participatory Design? In: Proc. PDC 2006, pp. 57-66. ACM (2006)

5. Braun, V., Clarke, V.: Using Thematic Analysis in Psychology. Qualitative Research in Psychology 3(2), 77-101 (2006)

6. Bucciarelli, L.: Delta Design Game. MIT Press (1991)

7. Buur, J., Soendergaard, A.: Video card game: an augmented environment for user centred design discussions. In: Proc. of DARE 2000, pp. 63-69. ACM (2000)

8. Dalsgaard, P., Halskov, K.: Reflective Design Documentation. In: Proc. of DIS 2012, pp. 428-437. ACM (2012)

9. Ehn, P.: Work-oriented Design of Computer Artifacts. Arbetslivscentrum (1988)

10. Everitt, K., Klemmer, S., Lee, R., Landay, J.: Two Worlds Apart: Bridging the Gap Between Physical and Virtual Media for Distributed Design Collaboration. In: Proc. CHI 2003, pp. 553-560. ACM (2003)

11. Fallman, D.: Design-Oriented Human-Computer Interaction. In: Proc. of CHI 2003, pp. 225-232. ACM (2003)

12. Flick, U.: An introduction to Qualitative Research. Sage Publishing (2006)

13. Geyer, F., Reiterer, H.: Experiences from Employing Evernote as a Tool for Documenting Collaborative Design Processes. In: Proc. of the DIS 2012 Workshop on Supporting Reflection in and on Design Processes, Newcastle, UK (2012)

14. Geyer, F., Pfeil, U., Budzinski, J., Höchtl, A., Reiterer, H.: AffinityTable - A Hybrid Surface for Supporting Affinity Diagramming. In: Campos, P., Graham, N., Jorge, J., Nunes, N., Palanque, P., Winckler, M. (eds.) INTERACT 2011, Part III. LNCS, vol. 6948, pp. 477-484. Springer, Heidelberg (2011)

15. Haller, M., Brandl, P., Leithinger, D., Leitner, J., Seifried, T., Billinghurst, M.: Shared Design Space: Sketching Ideas Using Digital Pens and a Large Augmented Tabletop Setup. In: Pan, Z., Cheok, D.A.D., Haller, M., Lau, R., Saito, H., Liang, R. (eds.) ICAT 2006. LNCS, vol. 4282, pp. 185-196. Springer, Heidelberg (2006)

16. Habraken, J., Gross, M.: Concept design games. Design Studies 9(3), 150-158 (1988)

17. Hartmann, B., Ringel Morris, M., Benko, H., Wilson, A.: Pictionaire: Supporting Collaborative Design Work by Integrating Physical and Digital Artifacts. In: Proc. of CSCW 2010, pp. 421-424. ACM (2010)

18. Iversen, O., Buur, J.: Design is a Game: Developing Design Competence in a Game Setting. In: Proc. of PDC 2002, pp. 22-28. CPSR (2002)

19. Iversen, O., Buur, J.: User Centred Design through the Keyhole: Video Design Case. In: Proc. of INTERACT 2003, pp. 431-438. IOS Press (2003) 
20. Kirk, D., Sellen, A., Taylor, S., Villar, N., Izadi, S.: Putting the Physical into the Digital: Issues in Designing Hybrid Interactive Surfaces. In: Proc. of BCS-HCI 2009, pp. 35-44. British Computer Society (2009)

21. Klemmer, S., Newman, M., Farrell, R., Bilezikjian, M., Landay, J.: The Designers' Outpost: A Tangible Interface for Collaborative Web Site Design. In: Proc. of UIST 2001, pp. 1-10. ACM (2001)

22. Löwgreen, J., Stolterman, E.: Thoughtful Interaction Design. MIT Press (2004)

23. Madsen, K., Dalsgaard, P.: Inspiration Card Workshops. In: Proc.of DIS 2006, pp. 2-11. ACM (2006)

24. Mendels, P., Frens, J., Overbeeke, K.: Freed: A System for Creating Multiple Views of a Digital Collection during the Design Process. In: Proc. of CHI 2011, pp. 1481-1490. ACM (2011)

25. Nakakoji, K., Yamamoto, Y., Matsubara, N., Shirai, Y.: Toward Unweaving Streams of Thought for Reflection in Professional Software Design. IEEE Software 29(1), 34-38 (2012)

26. Nakakoji, K., Yamamoto, Y., Takada, S., Reeves, B.: Two-Dimensional Spatial Positioning as a Means for Reflection in Design. In: Proc. of DIS 2000, pp. 145-154. ACM (2000)

27. Nelson, H., Stolterman, E.: The Design Way-Intentional Change in an Unpredictable World. Educational Technology (2003)

28. Rieber, L.P., Smith, L., Noah, D.: The value of serious play. Educational Technology 38(6), 29-37 (1998)

29. Rittel, H., Webber, M.: Dilemmas in a General Theory of Planning. Policy Sciences 4(2), 155-169 (1973)

30. Schön, D.: Designing as a Reflective Conversation with the Materials of a Design Situation. Research in Engineering Design 3, 131-147 (1992)

31. Schön, D.: Educating the Reflective Practitioner - Toward a New Design for Teaching and Learning in the Professions. Jossey-Bass Publishers (1987)

32. Schön, D.: The Reflective Practitioner. Basic Books (1983)

33. Sokoler, T., Edeholt, H.: Physically embodied video snippets supporting collaborative exploration of video material during design sessions. In: Proc. of NordiCHI 2002, pp. 139-148. ACM (2002)

34. Sokoler, T., Edeholt, H., Johansson, M.: VideoTable: a tangible interface for collaborative exploration of video material during design sessions. In: Ext. Abs. of CHI 2002, pp. 656-657. ACM (2002)

35. Swan, L., Tanase, D., Taylor, A.: Design's Processional Character. In: Proc. of DIS 2010, pp. 65-74. ACM (2010)

36. Törpel, B.: The design game in participatory design and design education: chances, risks and side effects. In: Proc. of PDC 2006, pp. 77-86. ACM (2006)

37. Turns, J.: Enhancing Design Education for Engineering Students: Supporting Planning, Tracking and Reflection. In: Proc. of ICLS 1996, pp. 593-594. International Society of the Learning Sciences (2006)

38. Vyas, D., Nijholt, A., Heylen, D., Kröner, A., van der Veer, G.: Remarkable: Objects: Supporting Collaboration in a Creative Environment. In: Proc. of UBICOMP 2010, pp. 37-40. ACM (2010)

39. Zimmerman, J., Forlizzi, J., Evenson, S.: Research through Design as a Method for Interaction Design Research in HCI. In: Proc. of CHI 2007, pp. 493-502. ACM (2007) 